TecnoHumanismo. Revista Científica

Junio - Agosto 2021

Volumen 1 / No.2

ISSN: $2710-2394$

pp. $346-360$

https://doi.org/10.53673/th.v1i11.77

https://tecnohumanismo.online

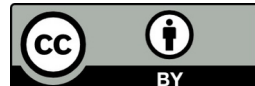

\title{
Servicio educativo, estilos de aprendizaje y formación profesional en los estudiantes de la especialidad de educación inicial del "Instituto de Educación Superior Pedagógico Público" - Huaraz, 2021
}

Educational service, learning styles and professional training in students of the initial education specialty of the "Public Pedagogical Higher Education Institute" - Huaraz, 2021

Atendimento educacional, estilos de aprendizagem e formação profissional em alunos da especialidade de educação inicial do "Instituto Público Pedagógico de Educação Superior" - Huaraz, 2021

\section{ARTÍCULO GENERAL}

\section{Flor Edith Paredes Diaz \\ paredesdfe@gmail.com \\ https://orcid.org/0000-0003-2516-1508 \\ Universidad Nacional Mayor de San Marcos, Lima - Perú}

Recibido 07 de Febrero 2021 | Arbitrado y aceptado 19 de Abril 2021 | Publicado en 28 Julio 2021

\section{RESUMEN}

Con el objetivo de identificar la relación entre el servicio educativo y los estilos de aprendizaje con la formación profesional de los estudiantes de la especialidad de educación inicial del Instituto de Educación Superior Pedagógico Público de Huaraz, 2021. Se desarrolló la presente investigación básica de diseño correlacional y transversal, para ello se planteó toda una estrategia para la recolección de los datos, donde las variables estudiadas: servicios educativos, estilos de aprendizaje y formación profesional fueron del tipo cuantitativo de medición nominal con codificación. Los datos obtenidos fueron de fuente primaria, recopilados mediante la aplicación de un cuestionario, previamente estructurado y valido por un panel de expertos. El cuestionario fue aplicado a una muestra de 103 estudiantes de la especialidad de Educación Inicial del "Instituto de Educación Superior Pedagógico Público" Huaraz. Los datos obtenidos fueron procesados mediante el uso programa SPSS versión 21 , para la prueba de hipótesis los datos fueron analizados con el estadístico Rho de Spearman, con un nivel de confianza de $95 \%$ y un margen de error del $5 \%$.

\section{ABSTRACT}

In order to identify the relationship between the educational service and the learning styles with the professional training of the students of the initial education specialty of the Public Pedagogical Higher Education Institute of Huaraz, 2021. The present basic research of correlational design was developed and cross-sectional, for this, a whole strategy for data collection was proposed, where the variables studied: educational services, learning styles and professional training were of the quantitative type of nominal measurement with coding. The data obtained was from a primary source, collected by applying a questionnaire, previously structured and validated by a panel of experts. The questionnaire was applied to a sample of 103 students of the specialty of Initial Education of the "Public Pedagogical Higher Education Institute" - Huaraz. The data obtained were processed using the SPSS version 21 program, for hypothesis testing the data were analyzed with the Spearman Rho statistic, with a confidence level of $95 \%$ and a margin of error of $5 \%$.

\section{RESUMO}

Com o objetivo de identificar a relação entre o serviço educacional e os estilos de aprendizagem com a formação profissional dos alunos da especialidade de educação inicial do Instituto Superior Pedagógico de Educação Pública de Huaraz, 2021. A presente pesquisa básica de desenho correlacional foi desenvolvida e transversal -secional, para isso, foi proposta toda uma estratégia de coleta de dados, onde as variáveis estudadas: serviços educacionais, estilos de aprendizagem e formação profissional foram do tipo quantitativo de medida nominal com codificação. Os dados obtidos foram de fonte primária, coletados por meio da aplicação de um questionário, previamente estruturado e validado por um painel de especialistas. $\mathrm{O}$ questionário foi aplicado a uma amostra de 103 alunos da especialidade de Educação Inicial do "Instituto Pedagógico Público de Ensino Superior" - Huaraz. Os dados obtidos foram processados no programa SPSS versão 21. Para teste de hipóteses, os dados foram analisados com a estatística Spearman Rho, com nível de confiança de $95 \%$ e margem de erro de $5 \%$. 
Los resultados evidencian que existe una buena relación $(\rho=0.919)$ entre el servicio educativo y formación profesional de los estudiantes. Denotándose que, el mal servicio educativo, mala infraestructura educativa, mala actuación del personal de la institución y la mala organización de la institución influye significativamente en tener una medianamente adecuada formación profesional de los estudiantes de la especialidad de educación inicial del instituto. De igual forma, se establece una buena relación $(\rho=0.617)$ entre los estilos de aprendizaje con la formación profesional en los estudiantes. Manifestandose que, la casi nunca aplicación de los estilos de aprendizaje reflexivo y pragmático y la a veces aplicación del estilo de aprendizaje activo y teórico influye significativamente en tener una medianamente adecuada formación profesional de los estudiantes. Por tanto, se concluye que existe una buena relación $(\rho=0.919)$ entre el servicio educativo y estilos de aprendizaje con la formación profesional de los estudiantes de la especialidad de Educación Inicial del Instituto de Educación Superior Pedagógico Público de Huaraz, donde el mal servicio educativo y la casi nunca aplicación de los estilos de aprendizaje influye significativamente en tener una medianamente adecuada formación profesional de los estudiantes.

Palabras clave: servicio educativo, estilos de aprendizaje, formación profesional.
The results show that there is a good relationship $(\rho=0.919)$ between the educational service and the professional training of the students. Denoting that, poor educational service, poor educational infrastructure, poor performance of the institution's staff and poor organization of the institution significantly influences having a moderately adequate professional training of students in the specialty of initial education of the institute. Similarly, a good relationship $(\rho=0.617)$ is established between learning styles and professional training in students. Manifesting that, the almost never application of reflective and pragmatic learning styles and sometimes the application of the active and theoretical learning style significantly influences having a moderately adequate professional training of students. Therefore, it is concluded that there is a good relationship ( $\rho=0.919)$ between the educational service and learning styles with the professional training of students of the specialty of Initial Education of the Public Pedagogical Higher Education Institute of Huaraz, where poor service Education and the application of learning styles almost never have a significant influence on having a moderately adequate professional training of students.

Keywords: educational service, learning styles, professional training.
Os resultados mostram que existe uma boa relação $(\rho=0,919)$ entre o atendimento educacional e a formação profissional dos alunos. Denotar que, atendimento educacional precário, infraestrutura educacional precária, desempenho insatisfatório do pessoal da instituição e má organização da instituição influenciam significativamente para que haja uma formação profissional moderadamente adequada dos alunos na especialidade de educação inicial do instituto. Da mesma forma, uma boa relação $(\rho=0,617)$ é estabelecida entre os estilos de aprendizagem e a formação profissional dos alunos. Manifestando isso, a quase nunca aplicação dos estilos de aprendizagem reflexivo e pragmático e a por vezes aplicação do estilo de aprendizagem ativo e teórico influencia significativamente na formação profissional moderadamente adequada dos alunos. Portanto, conclui-se que existe uma boa relação $(\rho=0,919)$ entre o atendimento educacional e os estilos de aprendizagem com a formação profissional de alunos da especialidade de Educação Inicial do Instituto Público de Ensino Superior Pedagógico de Huaraz, onde o atendimento ao Ensino é precário e a aplicação de estilos de aprendizagem quase nunca exerce influência significativa na formação profissional moderadamente adequada dos alunos.

Palavras-chave: serviço educacional, estilos de aprendizagem, formação profissional. 


\section{Introducción}

En la localidad de Huaraz, Ancash, existen pocas posibilidades en cuanto a la elección de una institución formadora de docentes de educación inicial, debido a que el Instituto superior Pedagógico es la única institución pública de toda la provincia que ofrece dicha especialidad. Por este motivo los estudiantes al momento de terminar sus estudios secundarios si deciden estudiar educación inicial deben elegir a dicha institución o en caso contrario una particular.

Es por ello que cuando el estudiante toma la decisión de iniciar sus estudios superiores en una determinada institución sea pública o privada, evalúa muchos aspectos para seleccionar la que pueda cumplir sus expectativas académicas, laborales, entre otras, es por ello, que las instituciones de educacion superior se esmeran en proporcionarle las herramientas necesarias para satisfacer esas motivaciones, sin embargo en la institución estudiada se está descuidando muchos de estos aspectos, impidiendo una buena formación profesional, aunque el presupuesto no es el mismo que manejaría una entidad privada, se evidenció que se está descuidando la organización administrativa, herramientas de enseñanza, entre otros.

Mazariegos (2006) señala que mientras existan más instituciones de nivel técnico estas se deberían esforzar en la calidad de los servicios que ofrecen desde la infraestructura, organización y comportamiento, sin descuidarse de los planes y objetivos específicos de la institución, para garantizar la satisfacción de los estudiantes.

Por su parte, Rodríguez y Vásquez (2012) refieren que los estilos se basan en las prácticas que los mismos estudiantes aplican para captar la información, sin embargo señalan que el análisis a fondo sobre un tema específico se está dejando de lado por el facilismo que las tecnologías de información ofrecen, por ello los docentes deben mantener el compromiso de ayudar a los estudiantes a identificar preferencias personales a la hora de organizar una actividad, planear estrategias para solucionar problemas cotidianos y transferir los conocimientos a otras situaciones similares.

Renés y Castro (2013) refieren que la formación profesional y técnica es parte importante del conjunto de procesos y actores sociales interrelacionados que constituyen un interés nacional, que deben responder a las exigencias de la competitividad de las empresas. En otras palabras, con el pasar del tiempo los estudiantes deben ir adquiriendo nuevos conocimientos que se ajusten más a las necesidades de la población.

Toda institución educativa es una organización que brinda servicios con la finalidad de satisfacer una serie de necesidades generadas por los estudiantes, el "Instituto de Educación Superior Pedagógico Público" Huaraz, tiene como misión: "Formar docentes de calidad, críticos, investigadores e innovadores; gestores del aprendizaje, comprometidos con una educación científica - humanística e intercultural, que contribuya al desarrollo sostenible del país". Para ello es necesario disponer de aulas adecuadas, docentes capacitados para la enseñanza, entre otros requerimientos que muchas veces se dejan de lado ya que se conforman con los implementos que tienen, desconociendo que la educación de hoy debe innovarse de 
acuerdo al avance del entorno social y económico, generando así un mercado laboral muy competitivo sin embargo solo accederá al puesto el egresado totalmente capacitado, si la formación profesional no fue la adecuada será difícil ingresar al mercado laboral.

Otro aspecto importante para la formación profesional es la forma como los estudiantes deben adquirir nuevos conocimientos, todos asimilamos información de forma diferente, es por eso que los docentes deben promover en ellos que no solo existe un único estilo de aprendizaje, debe motivar y capacitar sobre las distintas habilidades considerando la diversidad cognitiva de cada persona, es decir si el estudiante conoce más estilos de aprendizaje será más efectivo y enriquecedor académicamente.

Lo descrito anteriormente será abordado en el marco teórico que desarrolle el servicio educativo, estilos de aprendizaje y la formación profesional sobre para lo cual se seguirá el siguiente orden de investigación: En el primer capítulo, se refiere al planteamiento del estudio, la fundamentación, planteamiento del problema, objetivos, justificación, formulación de las hipótesis, identificación de las variables, metodología de la investigación y el glosario de términos. En el segundo capítulo trata sobre los antecedentes de la investigación y las bases teóricas. El tercer capítulo incluye la presentación, análisis e interpretación de los datos, asimismo cuenta con el proceso de prueba de hipótesis y la discusión de los resultados.

Finalmente se presenta las referencias bibliográficas, conclusiones, sugerencias y los anexos (matriz de consistencia, los instrumentos de recolección de datos, la validación por los expertos.

\section{Objetivo}

Identificar la relación entre el servicio educativo y los estilos de aprendizaje con la formación profesional de los estudiantes de la especialidad de Educación Inicial del Instituto de Educación Superior Pedagógico Público.

De igual forma se plantean los siguientes objetivos específicos:

- Analizar la relación entre el servicio educativo y formación profesional de los estudiantes.

- Establecer la relación entre los estilos de aprendizaje con la formación profesional en los estudiantes.

\section{Método}

Es una investigación básica de diseño correlacional y transversal. En esa línea se organizó toda una estrategia, para la recolección de los datos, donde las variables fueron del tipo cuantitativo de medición nominal con codificación. Los datos fueron de fuente primaria, obtenidos mediante la aplicación de un cuestionario, previamente estructurado y valido por un panel de expertos. El cuestionario fue aplicado a una muestra de 103 estudiantes de la especialidad de Educación Inicial del "Instituto de Educación Superior Pedagógico Público" - Huaraz. Los datos obtenidos fueron procesados mediante el 
uso programa SPSS versión 21, para la prueba de hipótesis los datos fueron analizados con el estadístico Rho de Spearman, con un nivel de confianza de 95\% y un margen de error del 5\%.

\section{Resultados}

\section{Servicio Educativo}

Figura 1: Valoración de las dimensiones del servicio educativo en los estudiantes de la especialidad de educación inicial del “Instituto De Educación Superior Pedagógico Público”-Huaraz.
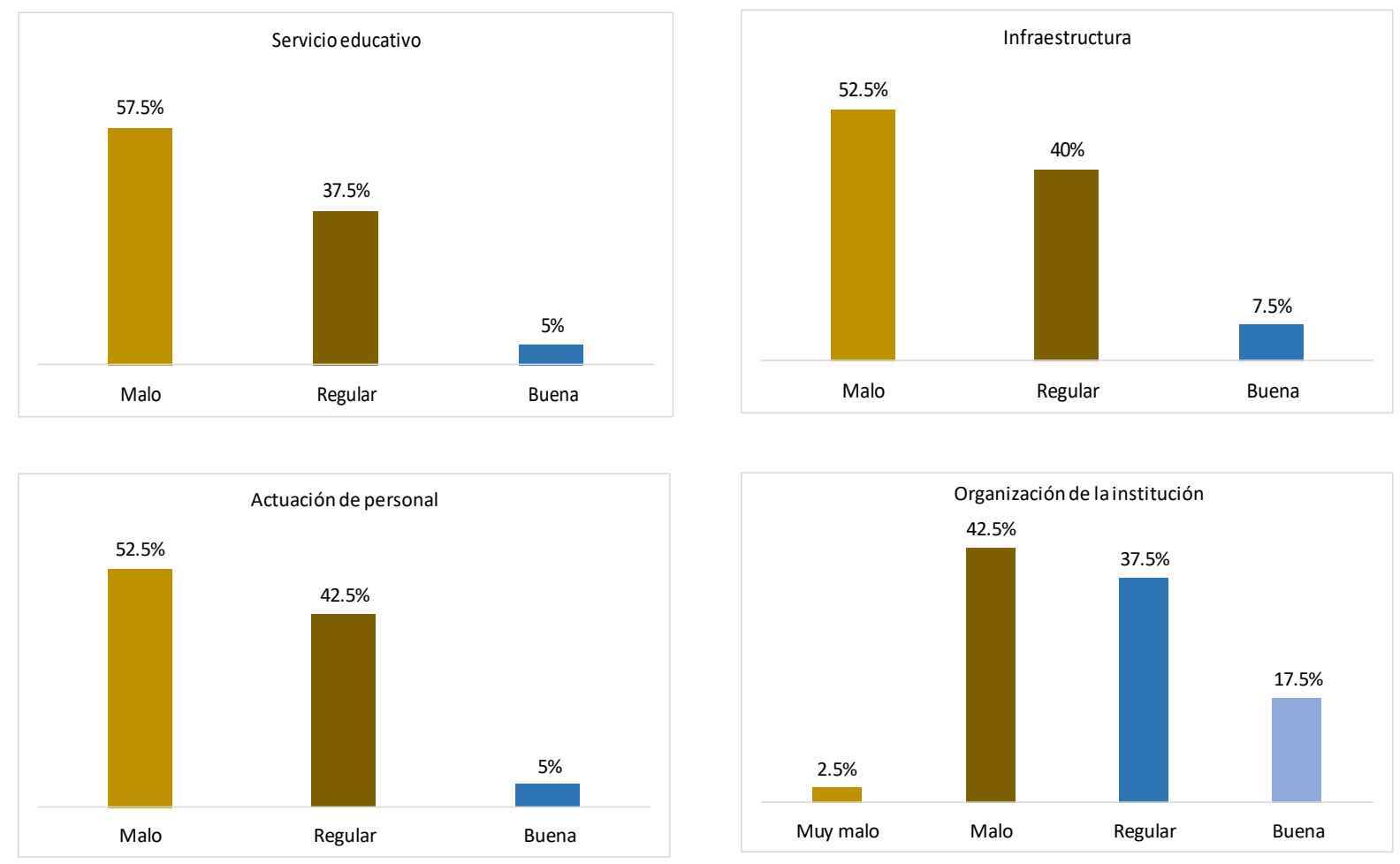

Como se observa en la figura 1 el $57.50 \%$ de los estudiantes de la especialidad de educación inicial del "Instituto De Educación Superior Pedagógico Público" de Huaraz, considera que el servicio educativo brindado por la institución es mala, mientras que el $37.5 \%$ la considera regular, y el 2\% como buena.

Respecto a la dimensión infraestructura, el 52.50\% considera a esta dimensión como malo, 40\% regular, y solo el 7.5\% como buena. En relación a la dimensión actuación del personal en la institución educativa, el 52.50\% considera como malo la infraestructura educativa, el 42.5\% regular, y solo el 5\% como buena. Semejantemente, el 42.50\% que la organización de la institución es mala, 37.5.5\% regular, $17.5 \%$ como buena y $2.5 \%$ muy malo. 
Flor Edith Paredes Diaz

Conforme a los resultados mostrados, el servicio educativo de la institución educativa estudiada es malo, donde puntualmente se considera que la infraestructura, actuación del personal y organización de la institución es mala.

\section{Estilos de aprendizaje}

Figura 2: Valoración de la aplicación del estilo de aprendizaje en los estudiantes de la especialidad de educación inicial del “Instituto De Educación Superior Pedagógico Público” - Huaraz.

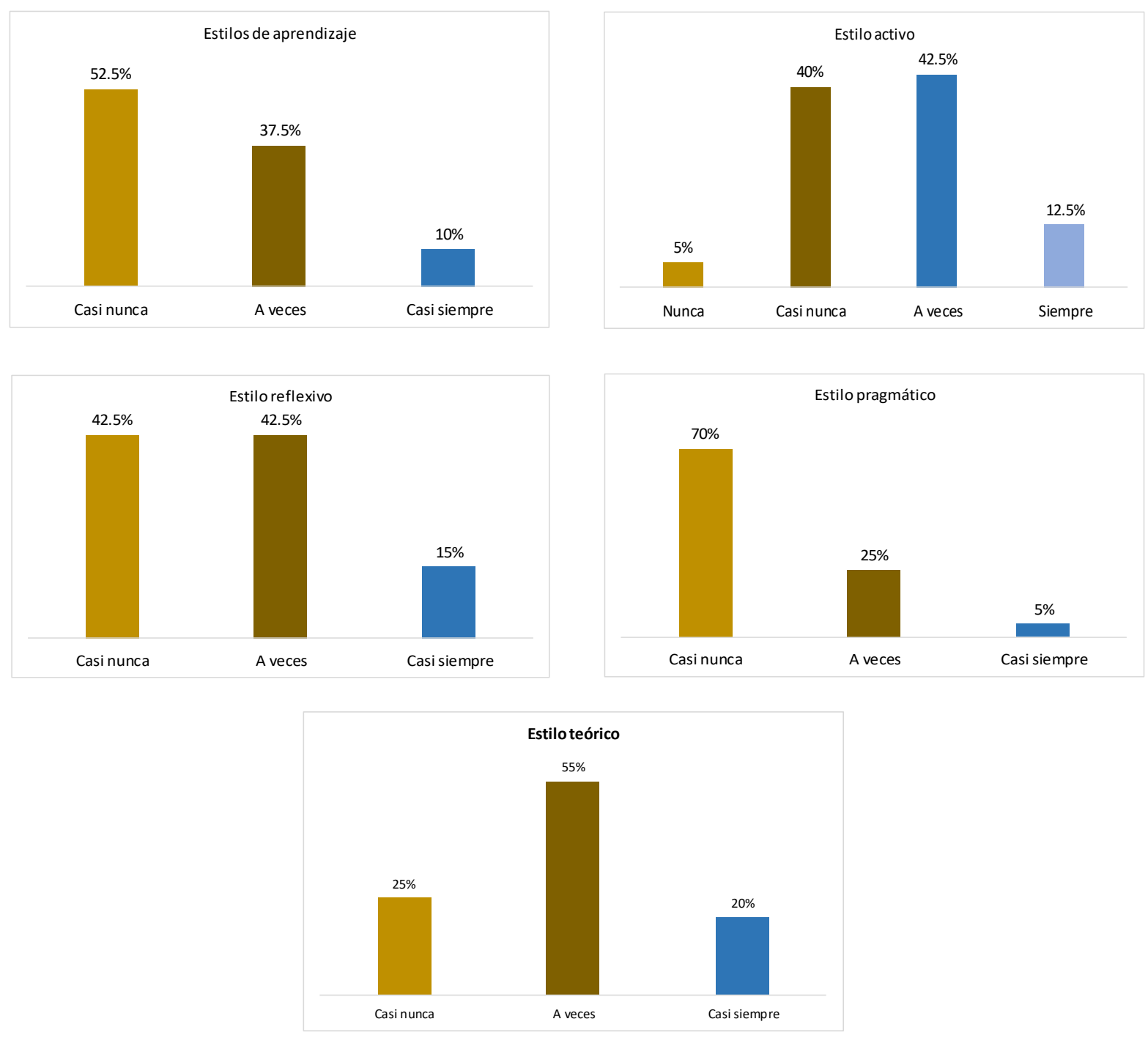

Como se observa en la figura 2, el $52.50 \%$ de los estudiantes de la especialidad de educación inicial del “Instituto De Educación Superior Pedagógico Público" de Huaraz, considera que casi nunca se emplean los estilos de aprendizaje, $37.5 \%$ a veces, y el $10.00 \%$ casi siempre. 


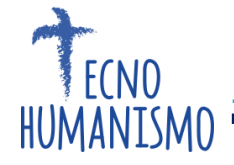

Flor Edith Paredes Diaz

Referente al estilo activo de aprendizaje, el $42.50 \%$ afirman que se utilizan a veces, el 40.0\% afirman que casi nunca se aplica este estilo, el $12.50 \% \%$ afirma que se utilizan siempre y el 5.00\% afirman casi nunca usar el estilo activo.

De igual forma, el $42.50 \%$ respondieron usar a veces el estilo reflexivo, el $42.5 \%$ responde casi nunca, y $15.00 \%$ mencionan emplear siempre. En referencia al estilo pragmático, el $70.00 \%$ respondieron utilizar casi nunca este estilo, 25\% a veces, el 5\% casi siempre. Finalmente, en referencia al estilo teórico, los datos demuestran que el $55.00 \%$ respondieron utilizar a veces, $25.00 \%$ casi siempre, y el $20.00 \%$ casi siempre.

De acuerdo a los resultados mostrados, los estudiantes de la especialidad de educación inicial del “Instituto De Educación Superior Pedagógico Público” de Huaraz, consideran que casi nunca se aplican o emplean los diferentes estilos de aprendizajes, esto evidencia una premisa negativa en la formación profesional de los mismos. 


\section{Formación Profesional}

Flor Edith Paredes Diaz

La figura 3, indica que el $52.50 \%$ de los estudiantes considera como a veces adecuado la formación profesional que recibe en el "Instituto De Educación Superior Pedagógico Público" de Huaraz, $40.00 \%$ poco adecuado, y el 7.50\% como adecuado. Puntualmente, en caso de los planes de estudios, el $57.50 \%$ de los estudiantes, también lo considera como a veces adecuado, $17.5 \%$ consideran como poco adecuado, y el 15.00\% como adecuado. Los resultados estadísticos mostrados confirman de acuerdo a la respuesta de la población estudiada el plan de estudios, según la opinión de la mayoría se encuentra entre a veces adecuado y poco adecuada, probamente repercutiendo en su formación profesional.
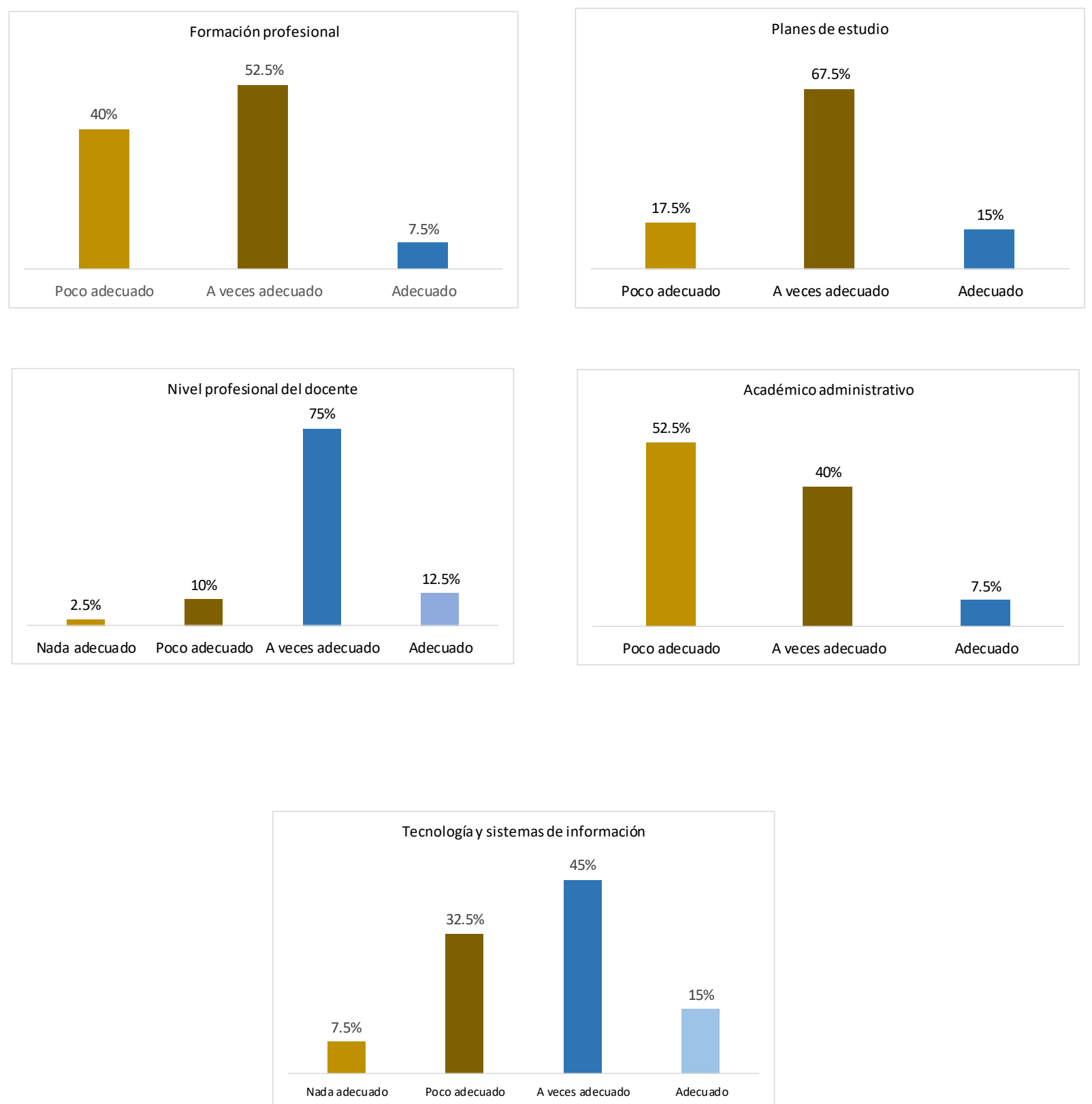

Figura 3: Valoración de las dimensiones de formación profesional en los estudiantes de la especialidad de educación inicial del “Instituto De Educación Superior Pedagógico Público” - Huaraz. 
Por otro lado, los resultados presentados en la figura 3, indican que el 75.00\% de los estudiantes considera como es a veces adecuado el nivel profesional del docente, el 12.5\% como adecuado, el 10.00\% adecuado y el $2.5 \%$ nada adecuado. De igual manera se denota que, el $52.50 \%$ de los estudiantes considera como poco adecuado el componente académico administrativo, $40.00 \%$ considera a veces adecuado, y el 7.50\% lo considera como adecuado. Finalmente, en caso de la tecnología e información, el $45.00 \%$ considera como a veces adecuado este aspecto, $32.5 \%$ poco adecuado, el $15.00 \%$ adecuado y $7.50 \%$ nada adecuado.

En general, los resultados mostrados para la formación profesional y sus dimensiones, son considerados como a veces adecuado o poco adecuado, tanto para la dimensión planes de estudio, nivel de formación docente, académico administrativo y la dimensión tecnología y sistemas de información. Entonces la formación profesional que cuenta la institución educativa no es suficiente, conforme la respuesta de la muestra la mayoría afirma o valora como a veces adecuado y poco adecuado el cual incide en el desarrollo de los profesionales.

\section{Relación entre el servicio educativo y estilos de aprendizaje con la formación profesional}

Tabla 1: Prueba Rho de Spearman ( $\alpha=0.05)$ para la relación entre el servicio educativo y estilos de aprendizaje con la formación profesional.

\begin{tabular}{|c|c|c|c|}
\hline & Correlaciones & & Formación profesional \\
\hline \multirow{6}{*}{ Rho de Spearman } & \multirow{3}{*}{$\begin{array}{c}\text { Servicio educativo - Estilos de } \\
\text { aprendizaje }\end{array}$} & $\begin{array}{l}\text { Coeficiente de } \\
\text { correlación }\end{array}$ & ,919 \\
\hline & & Sig. (bilateral) & 000 \\
\hline & & $\mathrm{N}$ & 103 \\
\hline & \multirow{3}{*}{ Formación profesional } & $\begin{array}{l}\text { Coeficiente de } \\
\text { correlación }\end{array}$ & $1,000 * *$ \\
\hline & & Sig. (bilateral) & . \\
\hline & & $\mathrm{N}$ & 103 \\
\hline
\end{tabular}

Para determinar la relación existente entre el servicio, educativo, estilos de aprendizaje con la formación profesional, se aplicó el estadístico de Rho de Spearman, que presenta un coeficiente de correlación muy buena, equivalente a 0.919 y el $\mathrm{p}=0,000$. Por lo cual, se rechaza la hipótesis nula y se acepta la hipótesis alterna; evidenciando que el servicio educativo y los estilos de aprendizaje se relacionan significativamente con la formación profesional en estudiantes de la especialidad de Educación Inicial del "Instituto de Educación Superior Pedagógico Público" - Huaraz.

Por tanto, se evidencia que el mal servicio educativo y la casi nunca aplicación de los estilos de aprendizaje influye significativamente en tener una medianamente adecuada formación profesional de los estudiantes de la especialidad de educación inicial del "Instituto De Educación Superior Pedagógico Público" de Huaraz. 


\section{Relación entre el servicio educativo con la formación profesional}

Tabla 2: Prueba Rho de Spearman ( $\alpha=0.05)$ para la relación entre el servicio educativo con la formación profesional.

\begin{tabular}{|c|c|c|c|}
\hline \multicolumn{3}{|c|}{ Correlaciones } & Formación profesional \\
\hline \multirow{6}{*}{$\begin{array}{c}\text { Rho de } \\
\text { Spearman }\end{array}$} & \multirow{3}{*}{ Servicio educativo } & Coeficiente de correlación &, 751 \\
\hline & & Sig. (bilateral) &, 000 \\
\hline & & $\mathrm{N}$ & 103 \\
\hline & \multirow{3}{*}{$\begin{array}{l}\text { Formación } \\
\text { profesional }\end{array}$} & Coeficiente de correlación & $1,000 * *$ \\
\hline & & Sig. (bilateral) & . \\
\hline & & $\mathrm{N}$ & 103 \\
\hline
\end{tabular}

Como se observa en la Tabla 2, al aplicar la fórmula de Rho de Spearman entre el servicio educativo con la formación profesional, con una significancia de 5\%, se halló un coeficiente de correlación buena de Rho $=0.751$ y el $\mathrm{p}=0,000$. Por lo cual se rechaza la hipótesis nula y se acepta la hipótesis alterna. Entonces, queda evidenciado que el servicio educativo se relaciona significativamente con la formación profesional en los estudiantes del X semestre del Instituto Superior Pedagógico Público de Huaraz.

Se denota que, el mal servicio educativo, mala infraestructura educativa, mala actuación del personal de la institución y la mala organización de la institución influye significativamente en tener una medianamente adecuada formación profesional de los estudiantes de la especialidad de educación inicial del "Instituto De Educación Superior Pedagógico Público" de Huaraz.

\section{Relación entre los estilos de aprendizaje con la formación profesional}

Tabla 3: Prueba Rho de Spearman $(\alpha=0.05)$ para la relación entre los estilos de aprendizaje con la formación profesional.

\begin{tabular}{|c|c|c|c|}
\hline \multicolumn{3}{|c|}{ Correlaciones } & Formación profesional \\
\hline \multirow{6}{*}{$\begin{array}{c}\text { Rho de } \\
\text { Spearman }\end{array}$} & \multirow{3}{*}{$\begin{array}{c}\text { Estilos de } \\
\text { aprendizaje }\end{array}$} & Coeficiente de correlación & ,617 \\
\hline & & Sig. (bilateral) &, 000 \\
\hline & & $\mathrm{N}$ & 103 \\
\hline & \multirow{3}{*}{$\begin{array}{l}\text { Formación } \\
\text { profesional }\end{array}$} & Coeficiente de correlación & $1,000 * *$ \\
\hline & & Sig. (bilateral) & . \\
\hline & & $\mathrm{N}$ & 103 \\
\hline
\end{tabular}

Al aplicar la fórmula de Rho de Spearman para evaluar la relación entre los estilos de aprendizaje con la formación profesional, con un margen de error al 5\%, se halló un coeficiente de correlación buena de Rho $=.617$ y el p = 0,000. Por lo tanto, se rechaza la hipótesis nula y se acepta la hipótesis alterna, que precisa que los estilos de aprendizaje se relacionan significativamente con la formación profesional en los estudiantes de la especialidad de Educación Inicial del "Instituto de Educación Superior Pedagógico Público" de Huaraz. 
Se evidencia que, el que la casi nunca aplicación de los estilos de aprendizaje reflexivo y pragmático y la a veces aplicación del estilo de aprendizaje activo y teórico influye significativamente en tener una medianamente adecuada formación profesional de los estudiantes de la especialidad de educación inicial del “Instituto De Educación Superior Pedagógico Público” de Huaraz.

\section{Discusión}

Esta investigación permitió desarrollar el objetivo planteado que es, conocer la relación entre el servicio educativo, estrategias de aprendizaje con la formación profesional en los estudiantes de la especialidad de Educación Inicial del "Instituto de Educación Superior Pedagógico Público" de Huaraz en el año 2021.

En general se determinó una correlación muy buena de Rho $=0.911$, entre las variables servicio educativo, estrategias de aprendizaje y la formación profesional. Se determinó, que el servicio educativo es considerado por el $50 \%$ de los estudiantes como malo y 52,5\% considera que casi nunca se aplica los estilos de aprendizaje. Por otro lado, a la formación profesional el 52.5\% lo refiere como a veces adecuado, seguido del $40.0 \%$ como poco adecuado. Con ello se pone en evidencia que entre el servicio que ofrece la institución de estudio casi nunca promueve la aplicación de estilos de aprendizaje de forma efectiva con cual incide en la insatisfacción del estudiante con respecto a su formación profesional, considerándolo como poco adecuado.

Siguiendo el mismo procedimiento estadístico, se determinó una correlación buena de Rho $=0.751$ entre la variable servicio educativo y la formación profesional de la población en estudio. Se encontró que el $57.3 \%$ de estudiantes califican de malo el servicio educativo brindado, seguido del $37.5 \%$ que lo califica como regular. Asimismo, el 52.5\% y 40.0\% consideran como a veces adecuado y poco adecuado la formación profesional brindada por la institución. Con lo cual se pone en evidencia, la necesidad de reorganizar y mejorar el servicio educativo como la infraestructura, personal y la organización, de lo contrario seguirá generando incidencias desfavorables para el desarrollo profesional de los estudiantes.

Resultados semejantes son reportados por Areche (2013), quien en su investigación destaca una relación significativa entre la gestión institucional y calidad del servicio educativo, puesto que los integrantes de la institución docentes y administrativos, el clima laboral, los planes de estudio permiten ganar una buena impresión del servicio, permitiendo una preparación idónea a las necesidades sociales. Por tanto, se ha comprobado que un buen servicio educativo es necesario para preparar a los estudiantes y estos alcancen sus objetivos profesionales. Po tanto, ellos necesitan de ambientes académicos adecuados y una organizacional adecuada a sus objetivos profesionales.

Por otro lado, entre las variables estilos de aprendizaje y formación profesional se encontró un coeficiente de correlación buena de Rho $=0,617$. Lográndose conocer la percepción de la aplicación de los estilos de aprendizaje, donde se evidencia que 52.5\% de estudiantes considera que casi nunca son utilizados, seguido de un 37.5\% que considera que a veces son utilizados los estilos de aprendizaje. En el caso de la formación profesional el 52.5\% y 40.0\% de los estudiantes, consideran como a veces adecuado y poco adecuado, respectivamente. Bajo esta premisa, se denota que se necesita promover los estilos de 
aprendizajes de carácter activo, reflexivo pragmático y teórico para que la adquisición de los nuevos conocimientos garantice la adecuada formación profesional de los estudiantes.

Contrariamente, De Torres (2013) analizó la relación de los estilos de aprendizaje con el rendimiento académico, encontrando que no mantienen relación alguna, ya que los estudiantes mantienen un promedio académico promedio. Sin embargo, manifiesta que los estilos de aprendizaje si tienen relación con el su entorno social - económico, debido a que los estudiantes de niveles socioeconómicos más bajos y de colegios municipales, tienden a poseer menores recursos para el estudio. Señalando que en cuestión la institución educativa debe proporcionar herramientas de aprendizajes y motivar en la aplicación de estilos de aprendizaje para una eficiencia en sus resultados académicos.

Respecto a la formación profesional Palop (2015) precisa que se debe incluir aspectos para desarrollo comunitario y personal. Coincidiendo con la presente investigación, ya que las instituciones educativas de nivel técnico deben formas profesionales en base a las necesidades laborales de la sociedad, la cual es cambiante y estas deben analizar el plan de estudios adecuado que los estudiantes estén capacitados para ingresar al mercado laboral que año tras año se vuelve más competitivo.

\section{Conclusiones}

1. Existe una buena relación $(\rho=0.919)$ entre el servicio educativo y estilos de aprendizaje con la formación profesional de los estudiantes de la especialidad de Educación Inicial del Instituto de Educación Superior Pedagógico Público de Huaraz, evidenciando que el mal servicio educativo y la casi nunca aplicación de los estilos de aprendizaje influye significativamente en tener una medianamente adecuada formación profesional de los estudiantes.

2. Existe una buena relación $(\rho=0.919)$ entre el servicio educativo y formación profesional de los estudiantes de la especialidad de Educación Inicial del Instituto de Educación Superior Pedagógico Público de Huaraz. Denotándose que, el mal servicio educativo, mala infraestructura educativa, mala actuación del personal de la institución y la mala organización de la institución influye significativamente en tener una medianamente adecuada formación profesional de los estudiantes de la especialidad de educación inicial del instituto en referencia.

3. Se establece una buena relación $(\rho=0.617)$ entre los estilos de aprendizaje con la formación profesional en los estudiantes de la especialidad de Educación Inicial del "Instituto de Educación Superior Pedagógico Público" de Huaraz. Se establece que, la casi nunca aplicación de los estilos de aprendizaje reflexivo y pragmático y la a veces aplicación del estilo de aprendizaje activo y teórico influye significativamente en tener una medianamente adecuada formación profesional de los estudiantes.

\section{Recomendaciones}


1. Al Instituto de Educación Superior Pedagógico Público” de Huaraz., se recomienda comprometerse con la generación de planes de mejora para el servicio educativo que incluyan el mejoramiento de la infraestructura, como la limpieza, acceso de las áreas de estudio, mayor comunicación entre las autoridades académicas, docentes y estudiantes. Del mismo modo, crear programas de capacitación específicos para el personal administrativo para mejorar su trato con el estudiante, asimismo reorganizar esta área para mayor efectividad en el tratamiento documentario.

2. A los docentes del instituto, se recomienda analizar el estilo predominante en el aula asignada para conocer las fortalezas y debilidades de los estudiantes al momento de adquirir nuevos conocimientos, con ello se podrá plantear y adaptar la metodología y los estilos de enseñanza idóneos a sus necesidades, del mismo modo comprometerse a su asesoramiento para su aplicación adecuada.

3. A las autoridades educativas del instituto, entablar mayor comunicación con los docentes al momento de crear los planes de estudio puesto que son ellos los que están en mayor contacto con los estudiantes y su realidad. Del mismo, el proceso de contratación debe tener un carácter más riguroso para garantizar la calidad profesional del docente dentro de las aulas, asimismo que tenga los conocimientos en cuanto a las TICS.

\section{Referencias Bibliograficas}

Boy Barreto, A. (2008). La gestión institucional y la calidad educativa en la institución educativa privada San Agustín de San Juan de Lurigancho -2007. Tesis de maestría UNE, 2008. Lima.

Díaz, E. (2012). Estilos de Aprendizaje. Revista Eídos, Edición Marzo - Agosto, Universidad Tecnológica Equinoccial, Ecuador.

Esquivel, N. y Huamani, D. (2016) Gestión administrativa y calidad de servicio educativo en las instituciones educativas privadas del nivel inicial del distrito de San Sebastián - Cusco, 2015 (Tesis pregrado) Universidad Andina Cusco, Cusco, Perú.

Fernández, S. (2010) "Prácticas y profesión en Trabajo Social: relaciones académicas y proceso metodológico". Cuadernos de Trabajo Social, 229-246.

Gutiérrez, A. S. (2005). Calidad en los servicios educativos. España: Díaz de Santos.

Lovelock, C. y Wirtz, J. (2009) Marketing de servicios. México: Editorial Pearson Educación

Mazariegos, C. (2006) El manejo del marketing en el servicio educativo. Universidad de San Carlos de Guatemala, Guatemala. Recuperado en: http://biblioteca.usac.edu.gt/tesis/08/08_1620_IN.pdf

Paz, R. (2005). Servicio al cliente: la comunicación y la calidad del servicio en la atención al cliente. España: Ideas propias Editorial S.L.

Pulido, D. (2006). Administración por Calidad. México: Limusa S.A. 


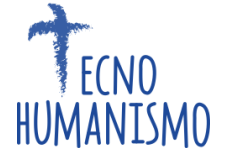

Servicio educativo, estilos de aprendizaje y formación profesional en los estudiantes de la especialidad de educación inicial del "Instituto de Educación Superior Pedagógico Público" - Huaraz, 2021

Renés, P. y A. Castro (2013) Análisis de la situación de la formación profesional desde el punto de vista de sus protagonistas. Educación XXI, Vol. 31, n².

Robbins, S. y Judge, T. (2009) Comportamiento organizacional. Decimotercera edición, Pearson Educación, México.

Rodríguez, M. y Vásquez, E. (2012) Fortalecer estilos de aprendizaje para aprender a aprender, UNED. Recuperado en: http://www2.uned.es/revistaestilosdeaprendizaje/numero 11/articulos/articulo 02.pdf

Sepúlveda, L. (2002). Algunas reflexiones acerca de los desafíos que presenta la formación académica de los Trabajadores Sociales en el Chile de hoy. Boletín Electrónico Surá.

Sovero, F. (2007). Cómo dirigir una Institución Educativa. AFA, Editores Importadores S.A. Lima.

Vicente, P. (2015) La formación profesional y su incidencia en el desarrollo local. Valencia, España. 